Horizons philosophiques

\title{
Le bien suprême est-il virtuellement réalisé dans le monde? Le jugement politique de Jacques Poulain
}

\section{Josette Lanteigne}

Volume 5, numéro 2, printemps 1995

Le philosophe et l'état

URI : https://id.erudit.org/iderudit/800981ar

DOI : https://doi.org/10.7202/800981ar

Aller au sommaire du numéro

Éditeur(s)

Collège Édouard-Montpetit

ISSN

1181-9227 (imprimé)

1920-2954 (numérique)

Découvrir la revue

\section{Citer cet article}

Lanteigne, J. (1995). Le bien suprême est-il virtuellement réalisé dans le monde? Le jugement politique de Jacques Poulain. Horizons philosophiques, 5(2), 74-83. https://doi.org/10.7202/800981ar d'utilisation que vous pouvez consulter en ligne. 


\title{
LE BIEN SUPRÊME EST-IL VIRTUELLEMENT RÉALISÉ DANS LE MONDE? LE JUGEMENT POLITIQUE CHEZ JACQUES POULAIN
}

\begin{abstract}
Carcequ'on nomme politique n'a pas plus de réalité qu'un cauchemar, même lorsqu'elle se traduit par des émeutes et des égorgements. Cette grosse bête, le canon, me tuera peut-être sans m'avoir convaincu qu'il existe, alors que quelques notes de Bach semblent soutenir le ciel. Que la lourdeur triomphe en Europe, ce ne sera jamais qu'une atroce apparence. Le vrai demeure hors de toute atteinte. (Julien Green, Joumal, 23 juin 1931)
\end{abstract}

Le jugement de Green en matière politique pourrait être partagé par Poulain. La politique actuelle est le pire des cauchemars, si elle a bien l'origine qu'il est possible de lui retrouver chez Kant ${ }^{1}$. Suivant celui-ci, si on a besoin de postuler un plus grand bienqui soit possible aussi parnotre collaboration, ce n'est pas par suite d'un manque de mobiles moraux pour faire le bien dans le monde, mais plutôt par manque de conditions extérieures dans lesquelles réaliser, conformément à ces mobiles, une fin en soi comme fin ultime morale ${ }^{2}$. On pourrait y voir une forme de surenchère. Ce n'est pas parce que les conditions extérieures ne supportent pas nos espérances que l'on doit en postuler de plus grandes encore dont les premières dériveraient. L'être humain a pourtant le devoir et le pouvoir de travailler à la réalisation d'une fin ultime morale, et la connaissance pragmatique explore "ce que l'homme, en tant

1. Dans la mesure où Kantest considéré pratiquement comme un saint en philosophie, lui attribuer ainsi la paternité même lointaine du cauchemar politique pourrait paraître relever de la provocation. Poulain ne pourrait cependant pas en être taxé, puisqu'il se réclame ouvertement des jugements synthétiques a priori (voir par ex. "Peut-on guérir de la politique?", Critique, t. XLIV, no 493-494 (1988), p. 507-530).

2. Kant, E., Oeuvres philosophiques, t. III, Paris, Éditions Gallimard, 1986, p. 257, note. On ne pourrait peut-être même pas former ces mobiles moraux, si on ne postulait un plus grand bien dont ils découlent. 
qu'être de libre activité, fait ou peut et doit faire de lui-même ${ }^{3}$ ». Or c'est précisément cette loi de la détermination ultime du vouloir qui engendre la capitalisation de l'action menant à la folie politique et à l'exploitation de l'homme par lui-même. Au terme de ce processus, l'homme contemporain se "primitivise" de plus en plus, tout en ordonnant sa nature sensible au bien suprême : l'action qui n'a d'autre fin qu'elle-même ${ }^{4}$. Cette "esthétisation" de la raison dans l'action serait en faitl'équivalent d'une sacralisation totémiste : "Cette sacralisation totémiste de la vie politique et son intériorisation dans la façon dont chacun y pense son rapport à soi et à autrui est indéniable, elle va jusqu'à servir de mesure à la morale elle-même $e^{5}$. La manière de suivre Kantn'est pas de "sensibiliser" la raison ${ }^{6}$, aboutissant à l'aberration d'une volonté de puissance qui juge sans concept, mais de revenir à l'origine théorique de la raison. La raison détermine la volonté, non en la sensibilisant ${ }^{7}$ mais en se déterminant. Je tenterai ici de montrer de quelle façon la raison se détermine elle-même chez Poulain en se déterminant comme dépositaire d'une raison théorique quasi infaillible dans le domaine de son exercice, qui est étendu à tout, et de quelle façon on peut répondre à cette prétention.

\section{Politique et jugement}

Le rapport politique est un rapport de force qui a pour enjeu la manipulation d'autrui. Le but est de le soumettre au bien suprême, aux dépens de sa liberté voire même de son existence ${ }^{8}$. Poulain ne suggère pas de se retirer complètement de ce rapport pour revenir à une raison pure théorique, mais de

3. Kant, E., Anthropologie du point de vue pragmatique, Paris, Vrin, 1964, p. 11.

4. Poulain, J., "Qu'est-ce que la politique?», p. 508-509.

5. Ibid., p. 509.

6. Poulain trouve le germe de cette fausse conception chez Kant, notamment dans son introduction à la Critique de la faculté de juger: "le concept de liberté a le devoir de rendre effectif dans le monde sensible la fin imposée par ses lois" (E. Kant, Oeuvres philosophiques, vol II, Paris, Gallimard, 1985, p. 929).

7. Poulain voit dans la sensibilisation l'équivalent de l'expérimentation scientifique, qui est récusée par lui comme expérience de vérité.

8. "Qu'est-ce que la politique?», p. 508. 
penser le rapport politique sous l'égide de la raison théorique (celle du jugement et de l'énonciation) plutôt que sous celle de la raison pratique, comme on l'a fait depuis Kant. Celui-ci soumettait la raison théorique à la raison pratique, et ni Apel ni Habermas ne l'ont contredit à ce sujet. Mais on aboutit à une situation où le consensus pratique ou même théorique ne dispose plus d'aucune objectivité. C'est ainsi que chez Habermas, la recherche du consensus finit par s'opposer à la recherche de l'objectivité :

...seul l'accord communicationnel peut produire [l'énonciation] comme telle, mais puisque cet accord ne peut être référé à une réalité autre que lui-même, rien ne semble permettre de le juger et de déceler la réalité ou l'irréalité du rapport d'interaction qu'il est censésanctionner comme tele.

Un autre problème, chez Habermas, c'est que «l'interaction communicationnelle s'avère être un rapport de force dont on ne peut se libérer qu'en ôtant, grâce à la réflexion, les contraintes unilatérales qu'il engendre ${ }^{10}$ ". Ce rapport de force est fondé sur l'aliénation de l'autre qui n'existe plus que pour obéir aux ordres qu'on lui donne, ou pour entériner le jugement de celui qui le soumet à sa volonté. La seule manière pour l'aliéné de se libérer est de se couper - s'il le peut - de tout rapport avec autrui. Mais ce faisant, il s'aliène de toute communication et par conséquent de tout rapport à la réalité :

\footnotetext{
S'émanciper, c'est dans ces conditions se déréaliser, s'abstraire de tous les rapports de langage dans lesquels on s'est reconnu, se faire savoir à soi-même qu'on est réellement autre que toutes les réalités avec lesquelles le langage a mis en rapport théorique ou pratique, conscient des opérations nécessaires àla production del'identification au langage et de ne se retrouver identique à aucune d'entre elles, totalement excentré"1.
}

9. Poulain, J., La neutralisation du jugement ou la critique pragmatique de la raison politique, Paris, Éditions de l'Harmattan, 1993, p. 224.

10. Ibid., p. 231.

11. Ibid., p. 232. 
Habermas saisit l'interaction communicationnelle sur le modèle des rapports de force, les rapports politiques, ce qui l'accule à une position paradoxale : le mouvement d'identification mutuelle au langage est compris à partir du mouvement d'inversion dialectique de ce rapport ${ }^{12}$. Mais si la philosophie d'Apel et d'Habermas se révèle ainsi inapte à nous faire sortir de la crise qu'ils ont été parmi les premiers à diagnostiquer, qui pourra suppléer à leur manque de jugement?

\section{La fin de «l'espoir pragmatique»}

L'homme contemporain a beau se primitiviser dans le rapport politique étendu à tout et à tous, c'est dans le prolongement de cette situation que naît l' "espoir pragmatique", qui fait croire en la réalité d'un rapport à l'autre irréductible au rapport à soi ${ }^{13}$. La pragmatique avait donné l'espoir de nous sortir des siècles de subjectivisme dans lesquels nous avait enfermés la philosophie de la conscience. Mais plutôt que d'espoir, c'est d' «échec pragmatique» qu'il faudrait parler : "L'identification anticipée de tous au jugement commun remplace et usurpe ici le jugement d'objectivité lui-même ${ }^{14}$ '). La pragmatique cherche dans la politique du consensus ce qu'elle doit précisément présupposer pour pouvoir le chercher; car elle doit présupposer le consensus objectif et réel, elle doit le penser vrai pour pouvoir le penser et l'imposer comme consensus réel. Une fois qu'il s'est reconnu lui-même, l'espoir pragmatique peut cesser de se rêver et la perversion contemporaine du rapport politique paraît curable. II suffit en effet d'inverser le rapport politique dans le rapport constitutif de l'être humain, le rapport du jugement :

L'exercice du jugement politique consiste en effet à ne se faire être et à ne faire être autrui que les seules réalités qu'on puisse être les uns pour les autres : celles qu'on a anticipé qu'on était pour pouvoir même les penser et qu'on se reconnaît être aussi effectivement qu'on a dû penser l'être, dès lors qu'on l'a pensé et qu'on s'y est identifié pour avoir pu y penser ${ }^{15}$.

12. Ibid., p. 231.

13. "Qu'est-ce que la politique?», p. 509.

14. Ibid., p. 523.

15. Ibid., p. 511. 
Autrement dit, on ne doit être que ce que l'on est les uns pour les autres, ce qu'on savait déjà. Ce jugement apparemment répétitif "guérit" de ce qu'il y a de folie dans le rapport politique, pour autant qu'on le saisit comme jugement philosophique. Le jugement philosophique (Poulain le définit comme un ujugement qui ne repose que sur son propre exercice, sur son affirmation et sur sa transmission ainsi que sur l'expérience qu'il rend luimême possible du seul fait qu'il parvienne à s'exprimer ${ }^{16}$ ") est la "thérapie" qui convient au jugement politique, en fixant au "seul besoin qu'il y a à combler pour combler tous les autres", à savoir "le besoin de juger ce qui rend la vie humaine possible ${ }^{17}$ ".

Pourtant, l'homme contemporain ne fait-il pas justement l'expérience de l'insuffisance chronique de son jugement de vérité? Le diagnostic de l'époque contemporaine a beau être aussi pessimiste qu'on voudra, ce qu'on veut savoir n'est-il pas si le malade finit par guérir? La réponse de Poulain à ces questions ne tient pas toujours compte du caractère «mortel» de la forme d'autisme ${ }^{18}$ qu'il a diagnostiquée :

Parce que son diagnostic comme tel révèle à lui seul que le seul besoin que puisse combler le discours est le besoin de vérité, parce que la façon dont il cherche à le combler n'est pas seulement inappropriée, mais fausse, il est possible de surmonter le mode autistique d'esthétisation de l'existence qu'il promeut ${ }^{19}$.

La justesse du diagnostic se démontre en invoquant le jugement même qui se trouve dénié20. II suffit donc de faire renaître le jugement qui a été mis entre parenthèses, pour redonner vie au malade. Ce qui renaît n'est pas le jugement d'une pensée abstraite toute-puissante, mais «le rapport dynamique d'identification ontologique engendré par le rapport

16. La neutralisation, p. 15-16.

17. "Qu'est-ce que la politique?", p. 529.

18. Cette notion sera explicitée plus loin au moyen d'exemples.

19. Poulain, J., L'Age pragmatique ou l'expérimentation totale, Paris, Éditions L'Harmattan, 1991, p. 181.

20. Ibid. 
communicationnel ${ }^{21}$ ". Toutefois, c'est cette même raison abstraite qui se perpétue dans la recherche du consensus argumentatif, qui est le jugement qui se fait au nom de tous au lieu d'un seul. User du jugement à la place d'un autre (ou de tous les autres) c'est en mésuser : «Plus les énonciateurs doivent parler au nom du monde visible ou au nom de la communauté, plus ils doivent mésuser de ce jugement, plus ils s'aperçoivent devoir le faire et plus ils sont portés à refouler l'exercice de ce jugement théorique exercé par délégation car ils le rendent responsable des malheurs qui leur arrivent ${ }^{22}$ ».

\section{Deux exemples d'inversion du jugement}

Dans L'Âge pragmatique, Poulain analyse deux situations où une forme de dénégation du jugement aboutit à un jugement objectif, sans qu'on prenne conscience de la contradiction performative qu'il y a à dénier le jugement tout en s'accordant la possibilité d'énoncer un jugement de vérité. Il s'agit de l'art et de la technique, deux formes de vie autonomes qui manifestent un autisme avancé tout en produisant des jugements de vérité. D'abord, l'art dit "abstrait», de l'expressionnisme à l'impressionnisme en passant par l'art cubiste, est autistique en voulant faire voir la vision elle-même : “...comme si l'on se voyait visuellement voir ce qu'on voit et la façon dont on voit ce qu'on voit : se faisant voir ainsi la coïncidence invisible d'un espace producteur de vision avecl'espace $v^{23}{ }^{23}$. La figuration de toutes les figurations possibles désoriente et désapprend la perception du réel (du monde, d'autrui, de soi). Il n'en reste pas moins que le jugement de vérité fait retour en esthétique, mais c'est un jugement tronqué : "L'expérience esthétique qui s'y rend accessible s'y objective telle qu'elle est et s'y juge être ce qu'elle est effectivement, même si elle ne constitue qu'une réalité tronquée, qu'une réalité qui s'est constituée comme telle en mettant entre parenthèses toute réalité ${ }^{24}$ ". 
La satisfaction infaillible que l'art fait éprouver tient à la façon dont il parasite la conscience technologique qui lui sert de support. L'identification au Tiers machine vient compléter le déni autistique du réel; l'homme contemporain trouve là son "essence» pragmatique, son ultime transformation: «il se transforme en l'être qui se répond à lui-même de façon nécessairement favorable et qui a conscience de le faire de façon aussi infaillible qu'il le doi ${ }^{25}$ ». Or cette idée de prendre sa base uniquement en soi n'est pas qu'un fantasme de la volonté de puissance, si l'être humain ne peut se faire être que ce qu'il se reconnaît être. Encore une fois, un jugement de vérité est attribuéà la prosopopée technologique, commeà la prosopopée iconique, mais le jugement y sert toujours uniquement à neutraliser toute possibilité réelle de jugement objectif. Non seulement les machines s'ordonnent-elles tout naturellement ${ }^{26}$ autour de nos besoins, sous la supervision de l'ordinateur, mais on peut considérer dès maintenant que le bien suprême est virtuellement réalisé dans le monde (puisqu'il suffirait d'étendre le mode de vie pragmatique des technologues à toute la planète pour en résoudre tous les maux). Le ridicule de ce jugement n'empêche pas qu'il soit difficile d'évaluer si la part du négatif (apathie, apraxie, agnosie, mort totale du stimulus) est plus grande que celle du positif (le jugement de vérité) chez Poulain. Car la conscience de vérité finit par se dénoncer elle-même :

\begin{abstract}
... en se faisant voir à elle-même, par les yeux mêmes de l'organisme qui la porte, qu'elle règle comme un objet les machines dans lesquelles elle a hypostasié et incorporé les actions motrices de l'organisme [...] en parvenant à transformer en machine sa conscience auto-régulatrice de conformité du produit réel par rapport au but visé, en parvenant à visibiliser cette conscience autonome dans l'ordinateur ${ }^{27}$.
\end{abstract}

25. Ibid., p. 207.

26. Il s'agit lci de la "seconde nature" de l'homme, de sa nature pragmatique (ibid., p. 208).

27. Ibid., p. 209. 
Qu'y a-t-il derrière l'art et la technique? La politique, diraiton; et Poulain ne serait pas le premier à la dénoncer, si tel était son propos. Mais il délaisse l'approfondissement des réalités politiques pour poursuivre son rêve de domination philosophique de la vie. Il est convaincu que cette domination est possible et qu'elle existe déjà au niveau transcendantal et théorique du langage ${ }^{28}$, qui ordonne intérieurement comme extérieurement toute réalité aussi bien que le ferait le programme animal le plus sophistiqué. C'est Gehlen qui a appris cette leçon à Poulain (une leçon que nous ne reprendrons pas dans son détail, car elle exige une étude beaucoup plus approfondie que celle qui a été entreprise ici). Poulain n'a pas retenu le conservatisme et le pessimisme de Gehlen, mais uniquement la preuve - faite sur le langage ${ }^{29}$ - que l'être humain est maître de son destin ${ }^{30}$.

\section{Politique ou jugement?}

Poulain tombe-t-il dans le défaut des modernes et des différentes pragmatiques, qui considéraient que l'homme peut se produire lui-même et instaurer ainsi les conditions de sa liberté? Par ailleurs, comment fait-il pour éviter de reproduire les indifférenciations qu'il relève chez les autres et en particulier chez Habermas?

28. La question du langage a été suffisamment galvaudée pour qu'on soit en droit d'attendre des précisions sur ce que l'auteur entend par cette expression. Si on fait abstraction du fait qu'il est toujours un peu absurde de parler du langage, on peut dire brièvement que Poulain entend toujours par là une réalité double; par ex. dans le langage parlé, il tient compte aussi bien de l'émission des sons que de leur réception; de méme, l'acte de prédication est étroitement lié à l'acte de référence, et ainsi de suite.

29. Seul le mode de reconnaissance de l'individu dans ce qu'il dit ou ce qui lui est dit (et non dans ce qui est fait) est essentiel (voir La Neutralisation, p. 83). En fin de parcours, Poulain présentera "l'échange communicationnel comme une épreuve de vérité dans laquelle c'est la reconnaissance mutuelle de l'identification mutuelle au vrai d'eux-mémes qui est dite comme vérité mutuelle et qui est la force transcendantale de la communication» (ibid., p. 244).

30. On sait que Gehlen ne partageait pas les thèses évolutionnistes de Darwin, puisqu'il affirme que l'homme a une position tout à fait particulière au sein de l'ensemble des êtres vivants. Au contraire des animaux qui sont préordonnés à leur environnement, le vivanthumain n'a pas de coordinations rigides préétablies avec son monde. C'est le langage qui prend la place du programme absent, et comme le langage est pour autant qu'on le sache limité aux êtres humains, on peut considérer qu'en un sens, ces êtres maîtrisent leur destin, à la différence des animaux qui ne font que répondre à des stimulus. 
Dans son dernier livre, La neutralisation du jugement, Poulain ne s'attaque pas pour l'essentiel à d'autres thèmes que ceux qui l'occupent depuis "Peut-on guérir de la politique?" et même avant. Mais plus on avance dans son œuvre, plus le genre de problème dont il traite dans différents contextes polémiques devient difficile à résoudre. Au début, il est facile de trouver la solution à son problème : le jugement de vérité qui avait été mis entre parenthèses doit être remis en exercice. Mais parvenu à l'étape finale de La neutralisation du jugement, il n'est plus possible de s'en sortir ainsi en donnant la formule magique. Pourtant, qu'il s'agisse du jugement politique, du jugement iconique ou du jugement technologique, ils parasitent tous le rapport de vérité, c'est-à-dire qu'ils attribuent à la politique, à l'art ou à la technologie ce qui revient à la vérité. Qu'est-ce que la vérité?

Pour obtenir la théorie positive de Poulain, il suffit peut-être de faire abstraction de sa critique négative. Ainsi, si Habermas méconnaît la spécificité du rapport d'identification mutuelle à la réalité de ce qui est dit, il suffirait de faire abstraction de cette méconnaissance pour recueillir son jugement de vérité. Mais l'indifférenciation que doitéviter Poulain estalors tout simplement celle qui identifierait son discours à celui d'Habermas. En effet, Poulain démontre qu'Habermas exclut la participation véritative de l'allocutaire. Mais en célébrant cette participation ${ }^{31}$, Poulain fait-il autre chose que de reprendre la position d'Habermas? Le problème que rencontre le lecteur critique de Poulain est qu'il exprime dans de nombreux passages quelque chose qui n'est pas foncièrement différent des thèses de Heidegger, Apel et Habermas. Et pourtant, alors que celles-ci sont défectueuses, le jugement de Poulain le serait moins. En vertu de quoi?

\section{Conclusion}

II s'avère que Poulain ne s'oppose pas autant que sa critique décapante aurait pu nous le faire croire, aux auteurs qu'il critique. Le domaine d'application de sa théorie s'étend 
effectivement à tout, mais il n'y a pas de fausse prétention dans le fait de demander que soit pensée vraie toute proposition et de dénoncer tous les cas où elle ne l'aura pas été, bien qu'elle ait dû se penser telle pour pouvoir être pensée. La contribution très personnelle de Poulain se réduit à peu de choses sur le plan du contenu. Par contre, il fournit une méthode pour libérer le jugement. Pour le jugement simpliciter, sa méthode se résume à ceci : le jugement (jugement politique, etc.) qui a été exprimé est-il aussi vrai qu'il a dû se penser vrai pour pouvoir se dire? Si on se fie à Poulain, il semble que le plus souvent, une telle question doive recevoir une réponse négative. On n'a pas besoin de s'en désoler outre mesure, puisqu'existe également le remède au mal : les jugements falsifiés par Poulain ne sontils pas récupérables (il suffirait de «restaurer en tout usage du langage le temps du jugement philosophique, qui ne repose que sur son propre exercice, sur son affirmation et sur sa transmission ainsi que sur l'expérience qu'il rend lui-même possible du seul fait qu'il parvient à s'exprimer ${ }^{32}$ "), alors que ceux qui sont laissés à eux-mêmes se trouvent falsifiés par la réalité? Sa thérapie philosophique semble donc tout indiquée à notre époque troublée, mais troublé, il ne l'est peut-être pas moins que nous. Le bien suprême est-il virtuellement réalisé dans le monde? La réponse actuelle de Jacques Poulain serait donc : "pas encore».

Josette Lanteigne 